\title{
Marketing social corporativo en el sector hostelero: una revisión sistemática
}

\author{
Corporate social marketing in the hostelry industry: \\ a systematic review
}

Gerard Costa Guix ${ }^{1}$, Mar Vila Fernández-Santacruz ${ }^{2}$, Susana Valdés ${ }^{3}$ ESADE, Universidad Ramon Llull, España

\section{RESUMEN}

Objetivos: Determinar la incidencia de las diferentes vertientes del marketing social corporativo en el sector hostelero. Métodos: Investigación realizada con una metodología cualitativa de revisión sistemática de las investigaciones previas. Resultados: Las peculiaridades del sector hostelero han sido ampliamente investigadas en términos de responsabilidad social empresarial; pero su potencial utilización como ventaja competitiva y las tipologías posibles de actuación estructuradas por varios autores en forma de marketing social corporativo son más recientes y desarrolladas en menor grado. Los principales resultados muestran que las actividades se centran en gran parte en el área de las prácticas empresariales socialmente responsables y en aquellas vinculadas a aspectos medioambientales, con un amplio espectro de actividades adicionales a realizar. Conclusiones: Las empresas del sector hostelero tienen la oportunidad y el desafío de utilizar sus actividades de responsabilidad social empresarial como una ventaja competitiva. Por esta razón, sin duda será necesario profundizar mediante investigaciones empíricas en el uso y los resultados de las políticas de marketing social corporativo en el sector hostelero en el futuro.

\begin{abstract}
Palabras clave: Sector hostelero, marketing social corporativo, responsabilidad social corporativa, ventaja competitiva, revisión sistemática.
\end{abstract}

gerard.costa@esade.edu sistemática. 


\section{ABSTRACT}

Objectives: To determine the incidence of different aspects about corporate social marketing in the hostelry sector. Methods: The research was realized with a qualitative methodology of previous research's systematic review. Results: The hostelry industry's peculiarities have been extensively investigated on corporate social responsibility terms; but its potential use as a competitive advantage and the possible action typologies structured by many authors in corporate social marketing form are newer and developed in lesser degree. The main results show that activities are focused largely on the business practices' area socially responsible and on which are linked to environmental aspects, with a broad spectrum of additional activities to realize. Conclusions: Companies in the hostelry industry have the opportunity and the challenge to use their activities of corporate social responsibility as a competitive advantage. For this reason, it will certainly be necessary to deepened through empirical research in the use and the corporate social marketing policies' results in the hostelry sector in the future.

Keywords: Hostelry industry, corporate social marketing, corporate social responsibility, competitive advantage, systematic review.

\section{INTRODUCCIÓN}

La responsabilidad social empresarial (RSE) es definida por Brown y Dancin (1) como las actividades de una compañía con respecto a sus obligaciones percibidas por la sociedad o como mínimo por sus grupos de interés, y es considerada por Porter y Kramer (2) como una inescapable prioridad para los líderes empresariales en todos los países.

La RSE ha sido investigada en diversos sectores, caso que resulta especialmente relevante en la industria del turismo y la hostelería por sus características: su visibilidad al escrutinio público, su impacto en transformar las localidades implicadas y el entorno natural, y la interrelación con los empleados y los residentes. Por ello el comportamiento socialmente responsable del sector turismo ha recibido una atención creciente desde la investigación en la pasada década, con trabajos específicos para el Perú como los de Valcuende y de De la Cruz (3) o Ficapal et al (4).

Una preocupación posterior ha sido investigar la relación entre la RSE de una empresa y su valor de mercado. Kotler (5) define el concepto societal marketing como la orientación al cliente que busca generar su satisfacción y su bienestar a largo plazo; y Drumwright y Murphy (6) definen el marketing social corporativo (MSC) como aquellas iniciativas de marketing que tienen al menos un objetivo no económico relacionado con el bienestar social y el uso de los recursos de la sociedad.

Reilly (7) sostiene que los consumidores deberían ver una compañía más favorablemente si soporta causas sociales, y así Maignan y Ferrell (8) identifican varios efectos positivos de los programas de responsabilidad social corporativa (RSC) en los consumidores.

Sen et al (9) detectan como ventajas de la RSE para las compañías los aumentos de los beneficios, la mayor lealtad de los clientes, o una mayor confianza y actitud positiva hacia la marca. Los beneficios que generan han sido analizados por ejemplo para el turismo comunitario en el Perú por Maldonado (10) o Sepúlveda et al (11).

El presente análisis ha detectado la oportunidad de revisar las investigaciones realizadas en el sector hostelería para obtener una categorización de los casos investigados de uso de la RSE y así aumentar el valor de mercado de una compañía. Las categorías las definiremos sobre la base de dos trabajos reconocidos: la clasificación de Kotler (12), y la específica del sector hostelero de Holcomb et al (13). 


\section{MATERIAL Y MÉTODOS}

El método de revisión sistemática tiene su origen en la comunidad médica, y como documentan Denyer y Neely (14) o Tranfield et al (15), posteriormente ha sido adoptada en las ciencias sociales y la investigación sobre la gestión empresarial. Se ha seguido el proceso de tres etapas: planificación, conducción y evaluación.

El objetivo de la planificación ha sido acumular el máximo de estudios basados en evidencias, y se ha consultado a un panel multidepartamental de la Universidad Ramon Llull (URL): Marketing, Ciencias Sociales, Política de Empresa; Instituto de Innovación Social y GRUGET (Grupo de Gestión de Empresas Turísticas). Como resultado obtuvimos una primera identificación desde todas las áreas implicadas de los términos clave a utilizar.

La conducción se realizó con búsquedas en cuatro bases de datos académicas relevantes (Emerald, EBSCO, Ingenta y ProQuest); complementada con búsquedas manuales en journals académicos siguiendo el ranking sectorial de turismo de Law y Van der Veen (16), y con la perspectiva profesional obtenida al consultar la base WARC. El resultado final fue la obtención de 85 investigaciones referidas al uso del marketing social corporativo en el sector hotelero.

La evaluación de la calidad se aplicó con tres criterios: máximo número de citas en Google Académico, las publicaciones más recientes y finalmente aquellas procedentes de publicaciones no especializadas en la RSE.

Con este criterio combinado acabamos aceptando una revisión de 13 investigaciones. Se ha extraído y sintetizado los datos mediante una codificación a través del software Atlas.Ti y el uso de las categorías propuestas por Kotler (12) y Holcomb et al (13).

\section{RESULTADOS}

El método de revisión sistemática ha permitido obtener 13 trabajos empíricos, listados en la tabla $N^{0} 1$, los cuales se analizaron siguiendo las categorizaciones de referencia, para obtener una mejor comprensión de las áreas que han sido más identificadas y cuáles quedan pendientes de profundizar.

Holcomb et al (13) consideran cinco grandes áreas de RSE utilizables en marketing social corporativo. Las políticas medioambientales son las más presentes, área que impulsa la proactividad medioambiental, una diferenciación del producto en la industria hotelera. Las demás políticas consideradas han aparecido preferentemente desde el 2005, y están vinculadas a las relaciones con la comunidad local más allá de la simple filantropía, a las relaciones con el equipo de trabajadores, al mercado y a la relación con los valores y visión de la propia organización.

Kotler (12) define seis subcategorías de actividades de marketing social corporativo. Las predominantes son la filantropía corporativa, en forma de donaciones caritativas, que está presente hasta en el $80 \%$ de los hoteles investigados; y las prácticas empresariales socialmente responsables, como la gestión de residuos, compras o uso de energía. También hay diversas prácticas detectadas en promoción de causa, con empresas que incentivan que empleados y clientes apoyen causas locales, programas corporativos de asociación con organizaciones de caridad; y en voluntariado corporativo, que motiva a los empleados a realizar voluntariado en la comunidad. Finalmente, hay dos categorías sin actividades detectadas: el marketing con causa y el marketing social corporativo.

Como resultado final, todos los artículos identificados han sido analizados en las áreas estudiadas, y clasificados sobre la base de ambos criterios. 
Tabla $N^{\circ}$ 1: Categorización de artículos identificados y revisados con la metodología

\begin{tabular}{|c|c|c|c|}
\hline Códigos Kotler & \multicolumn{3}{|c|}{$\begin{array}{l}\text { PESR: Prácticas empresariales socialmente responsables } \\
\text { FS: Filantropía corporativa } \\
\text { PC: Promoción de causa } \\
\text { VC: Voluntariado corporativo }\end{array}$} \\
\hline $\begin{array}{l}\text { Códigos } \\
\text { Holcomb }\end{array}$ & \multicolumn{3}{|l|}{$\begin{array}{l}\text { MA: Medioambiente } \\
\text { C: Comunidad } \\
\text { M: Mercado } \\
\text { ET: Equipo de trabajo } \\
\text { W: Visión y Valores }\end{array}$} \\
\hline Artículos & Área estudiada & Kotler & Holcomb \\
\hline Han et al (17) & $\begin{array}{l}\text { Protección medioambiental, gestión de residuos, } \\
\text { compras, uso de la energía, prácticas de } \\
\text { conservación }\end{array}$ & PESR & MA \\
\hline $\begin{array}{l}\text { bohdanowicz y } \\
\text { Zientara (18) }\end{array}$ & $\begin{array}{l}\text { Calidad de vida en la comunidad huesped, } \\
\text { donaciones, caridad, compra comercio justo y } \\
\text { bienestar de los empleados }\end{array}$ & $\begin{array}{l}\text { PESR } \\
\text { FC } \\
\text { PC } \\
\text { VC }\end{array}$ & $\begin{array}{l}\text { MA } \\
C \\
M \\
E T\end{array}$ \\
\hline Wildes (19) & $\begin{array}{l}\text { Iniciativas de medioambiente y de sostenibilidad } \\
\text { RSE en la estrategia corporativa. Soporte a la } \\
\text { comunidad local y cultura, comercio justo. }\end{array}$ & $\begin{array}{l}\text { PESR } \\
\text { FC }\end{array}$ & $\begin{array}{l}\text { MA } \\
C\end{array}$ \\
\hline $\begin{array}{l}\text { Claver Cortés et } \\
\text { al (20) }\end{array}$ & Medio ambiente & PESR & MA \\
\hline Kasim (21) & Medio ambiente & PERS & MA \\
\hline Ayuso (22) & Medio ambiente, iniciativas sostenibilidad & PESR & MA \\
\hline Holcomb et al (13) & $\begin{array}{l}\text { Donaciones, caridad, programa voluntariado } \\
\text { empleados, políticas de diversidad hacia socios. }\end{array}$ & $\begin{array}{l}\text { PESR } \\
\text { FC } \\
\text { VC }\end{array}$ & $\begin{array}{l}C \\
M \\
E T \\
M A \\
W V\end{array}$ \\
\hline $\begin{array}{l}\text { Manakttola y } \\
\text { Jauhari (23) }\end{array}$ & Medio ambiente & PESR & MA \\
\hline $\begin{array}{l}\text { Erdogan y Baris } \\
(24)\end{array}$ & $\begin{array}{l}\text { Protección medioambiental, gestión de residuos, } \\
\text { compras, uso energía, prácticas de conservación. }\end{array}$ & PESR & MA \\
\hline $\begin{array}{l}\text { Van der Merwe } \\
\text { y Wocke (25) }\end{array}$ & $\begin{array}{l}\text { Medioambiente. Nivel de implantación de RSC y } \\
\text { prácticas responsables. }\end{array}$ & $\begin{array}{l}\text { PESR } \\
\text { FC } \\
\text { PC }\end{array}$ & $\begin{array}{l}\text { MA } \\
C \\
M \\
\text { V }\end{array}$ \\
\hline $\begin{array}{l}\text { Ashley y } \\
\text { Haysom (26) }\end{array}$ & $\begin{array}{l}\text { Filantropía, medioambiente y soporte a } \\
\text { comunidad local }\end{array}$ & $\begin{array}{l}\text { PESR } \\
\text { FC }\end{array}$ & $\begin{array}{l}\text { MA } \\
C\end{array}$ \\
\hline Jones et al (27) & $\begin{array}{l}\text { Mercado puestos de trabajo, medioambiente y } \\
\text { comunidad. }\end{array}$ & $\begin{array}{l}\text { PESR } \\
\text { PC } \\
\text { FC }\end{array}$ & $\begin{array}{l}\text { MA } \\
\text { C } \\
\text { M } \\
\text { ET }\end{array}$ \\
\hline Bohdanowicz (28) & Medioambiente & PESR & MA \\
\hline
\end{tabular}




\section{DISCUSIÓN}

Las investigaciones precedentes han detectado dos grandes áreas de prácticas empresariales en marketing social corporativo que busquen generar ventajas competitivas desde el atractivo social de una empresa en hostelería: aquellas vinculadas a la gestión medioambiental, y las prácticas de negocio socialmente responsables. Hemos analizado cómo en el resto de áreas potenciales hay oportunidades de desarrollar actividades de RSE que sean utilizadas para aumentar el valor de mercado, tal y como está ocurriendo en otros sectores.

La primera gran actividad que ya se está desarrollando es la filantropía corporativa, que ha evolucionado de la simple caridad a una actividad que implica al resto de grupos de interés, sean empleados o clientes.

El segundo grupo de actividades que crecerán en el sector hostelero, debido a sus peculiaridades específicas, son los aspectos vinculados a fuerza de trabajo, impacto en la comunidad y mercado local. Aún y habiendo identificado cómo son pequeños los segmentos de clientes interesados en estas actividades, hay sin duda una posibilidad de crear ventajas competitivas mediante acciones socialmente responsables en estos ámbitos. El último reto para el sector será desarrollar las actuaciones todavía incipientes vinculadas a la visión y valores de la organización, aquellas generadas desde el propio posicionamiento de RSE.

Una recomendación para futuras investigaciones es profundizar en el efecto del marketing social corporativo sobre el consumidor final. Adicionalmente a los trabajos de Lafferty y Goldsmith (29), y de Sen et al (9), resulta pertinente también profundizar sobre cuál es el impacto diferencial de cada una de las categorías de actividades del marketing social corporativo que han sido investigadas, cuáles son preferidas, o si generan comportamientos como un aumento de la fidelidad o de la recomendación boca en boca.

\section{REFERENCIAS BIBLIOGRÁFICAS}

1. Brown TJ, Dacin PA. The company and the product: corporate associations and consumer product responses. The Journal of Marketing. 1997: 68-84.

2. Porter M, Kramer MR. Strategy \& Society. The link between competitive advantage and corporate social responsibility. Harv Bus Rev. 2006; december:78-92.

3. Valcuende del Río JM, De la Cruz Quispe L. Turismo, poblaciones locales y organizaciones no gubernamentales: un análisis de caso en Madre de Dios (Perú). Comité Editorial Director: Agustín Santana Talavera 2009; 7:179. (4. Ficapal J, Guix M, Caller F, Pamies MA. Turismo responsable y desarrollo humano, caso práctico en Perú.

5. Kotler P. What Consumerism Means for Marketers. Harvard Business Review 1972; 50: 48-57.

6. Drumwright $M$, Murphy E. In: Bloom PN, Gundlach GT, editors. Corporate Societal Marketing; Handbook of Marketing and Society Thousand Oaks, CA: Sage Publications; 2001. p. 162183.

7. Reilly J. Charitable Works Sells at a Number of Firms. Marketing News 2000 09:46.

8. Maignan I, Ferrell OC. Corporate Social Responsibility and Marketing: An Integrative Framework. Journal of the Academy of Marketing Science 2004 / Winter;32(1):3-19.

9. Sen S, Bhattacharya CB, Korshun D. The role of corporate social responsibility in strengthening multiple stakeholder relationships: a field experiment. Journal of the Academy of Marketing Science 2006;34:158-166.

10. Maldonado C. Pautas metodológicas para el análisis de experiencias de turismo comunitario. International Labour Office; 2005.

11. Sepúlveda H, Basurto R, Vizcarra $Y$. Plan estratégico para el desarrollo del turismo rural comunitario en la región Cusco 2013.

12. Kotler P/,N. Corporate Social 
Responsibility: Doing the Most Good for Your Company and Your Cause. Hoboken, NJ: John Wiley \& Sons; 2005.

13. Holcomb JL, Upchurch RS, Okumus F. Corporate social responsibility: what are top hotel companies reporting? International Journal of Contemporary Hospitality Management 2007; 19(6):461-475.

14. Denyer D, Neely A. Introduction to special issue: innovation and productivity performance in the UK. International Journal of Management Reviews 2004;5(3-4):131-135.

15. Tranfield D, Denyer D, Smart P. Towards a methodology for developing evidence informed management knowledge by means of systematic review. Br J Manage 2003; 14(3):207-222.

16. Law R, Van der Veen R. The popularity of prestigious hospitality journals: a Google Scholar approach. International Journal of Contemporary Hospitality Management 2008;20(2):113-125.

17. Han H, Hsu LJ, Sheu C. Application of the theory of planned behavior to green hotel choice: Testing the effect of environmental friendly activities. Tourism Management 2010;31(3):325334.

18. Bohdanowicz P, Zientara P. Hotel companies' contribution to improving the quality of life of local communities and the well-being of their employees. Tourism \& Hospitality Research 2009 $02 ; 9(2): 147-158$.

19. Wildes VJ. How can organizational leaders really lead and serve at the same time? International Journal of Contemporary Hospitality Management $200801 ; 20(1): 67-78$.

20. Claver-Cortés E, Molina-Azorín JF, Jorge Pereira-Moliner, López-Gamero MD. Environmental Strategies and Their Impact on Hotel Performance. Journal of Sustainable Tourism 2007 11; 15(6):663-679.

21. Kasim A. Corporate environmentalism in the hotel sector: evidence of drivers and barriers in Penang, Malaysia. Journal of Sustainable Tourism 2007; 15(6):680-699.

22. Ayuso S. Comparing voluntary policy instruments for sustainable tourism: The experience of the Spanish hotel sector. Journal of Sustainable Tourism 2007;15(2): 144-159.

23. Manaktola K, Jauhari V. Exploring consumer attitude and behaviour towards green practices in the lodging industry in India. International Journal of Contemporary Hospitality Management 2007; 19(5):364-377.

24. Erdogan N, Baris E. Environmental protection programs and conservation practices of hotels in Ankara, Turkey. Tourism Management 2007; 28(2):604614.

25. Van der Merwe M, Wocke A. An investigation into responsible tourism practices in the South African hotel industry. 2007.

26. Ashley C, Haysom G. From philanthropy to a different way of doing business: strategies and challenges in integrating pro-poor approaches into tourism business. Development Southern Africa 2006; 23(2):265-280.

27. Jones P, Comfort D, Hillier D. Reporting and reflecting on corporate social responsibility in the hospitality industry: A case study of pub operators in the UK. International Journal of Contemporary Hospitality Management 2006; 18(4):329-340.

28. Bohdanowicz P. European Hoteliers' Environmental Attitudes Greening the Business. Cornell Hotel \& Restaurant Administration Quarterly 2005;46(2): 188-204.

29. Lafferty BA, Goldsmith RE. Causebrand alliances: does the cause help the brand or does the brand help the cause. Journal of Business Research 2005; 58(423-431). 